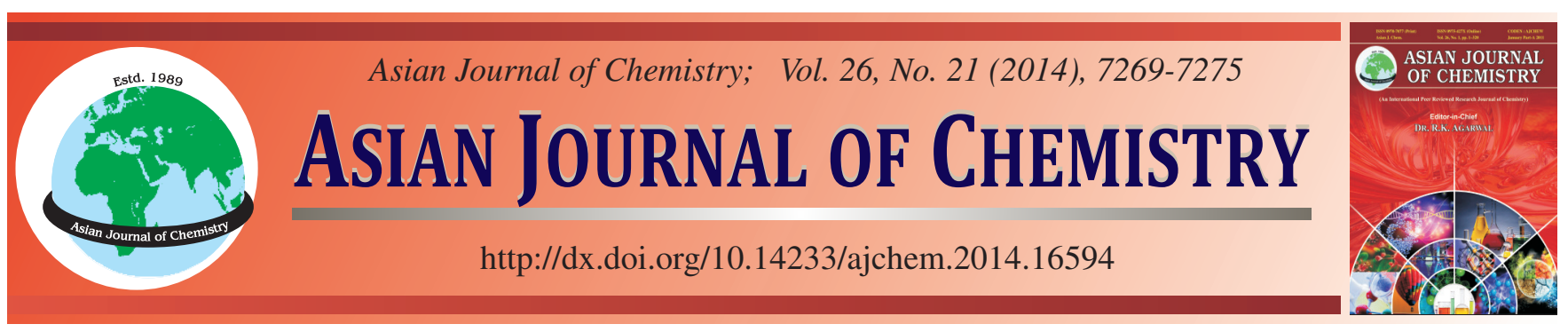

\title{
Design, Synthesis and Activity Evaluation of N-(pyridin-4-yl) Salicylamides as Antimycobacterial Agents
}

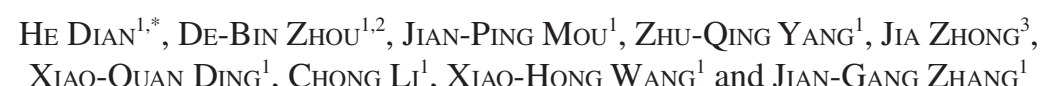

${ }^{1}$ Institute of Medicinal Chemistry, School of Pharmacy, Lanzhou University, Lanzhou 73000, P.R. China

${ }^{2}$ The First Hospital of PLA, Lanzhou 730030, P.R. China

${ }^{3}$ Department of Pharmacy, Pulmonary Hospital of Lanzhou, Lanzhou 730046, P.R. China

*Corresponding author: Fax: +86 931 8915686; Tel: +86 13008738922; E-mail: hed@1zu.edu.cn

A series of N-(pyridin-4-yl) salicylamides derivatives were prepared through acylation of the corresponding acetylsalicyloyl chlorides with substituted 4-amino-pyridines. These compounds were evaluated in vitro for antimycobacterial activities against Mycobacterium tuberculosis (TB) and Mycobacterium avium (A) by the minimum inhibitory concentrations (MIC) values. Eight of the compounds exhibited lower MIC against A than the one of isoniazide. Meanwhile, four of the compounds exhibited good anti-TB activity compared to isoniazide. Antimycobacterial activities of N-(pyridin-4-yl) salicylamides were influenced by the balance between hydrophobicity and electron withdrawing substituent effects on the phenyl and pyridine ring. These studies show that the compounds might serve as prospective wide-spectrum antimycobacterial substances.

Keywords: Mycobacterium tuberculosis, N-(pyridin-4-yl) salicylamides, Activity evaluation, Synthesis.

\section{INTRODUCTION}

Searching for new antimycobacterial compounds is one of the most challenging tasks in current medicinal chemistry. Particularly, antimycobacterial properties of salicylanilides ${ }^{1,2}$ inhibiting two-component regulatory systems in mycobacteria, as a known strategic opportunity for design of selective antimycobacterial agents ${ }^{1-8}$, is of great interest. Isosteres of salicylanilides were systematically documented, i.e. 3-hydroxypicolinanilides $^{9}$, 2-sulfanylbenzanilides ${ }^{9}$, N-benzylsalicylamides ${ }^{10}$ and N-pyridin-2-ylsalicylamides ${ }^{11}$. However, Npyridin-4-ylsalicylamides as antimycobacterial agents have rarely been studied systematically in literatures. We thus prepared a series of new N-pyridin-4-yl salicylamide analogues (Fig. 1) to study their antimycobacterial activities by varying the substituents on the heterocyclic and salicylic moieties.

The synthetic route to $\mathrm{N}$-(pyridin-4-yl) salicylamides was shown in Fig. 1. N-(pyridin-4-yl) salicylamides 1a-6h were prepared by treatment of the corresponding substituted acetylsalicyloyl chlorides with substituted 4-amino-pyridines in chloroform in the presence of triethylamine. After addition of $10 \%$ hydrochloric acid and saturated sodium bicarbonate the products was isolated in more than $85 \%$ yield according to the theoretical value. The structure was fully identified by ${ }^{1} \mathrm{H}$ NMR, ${ }^{13} \mathrm{C}$ NMR and IR spectra.

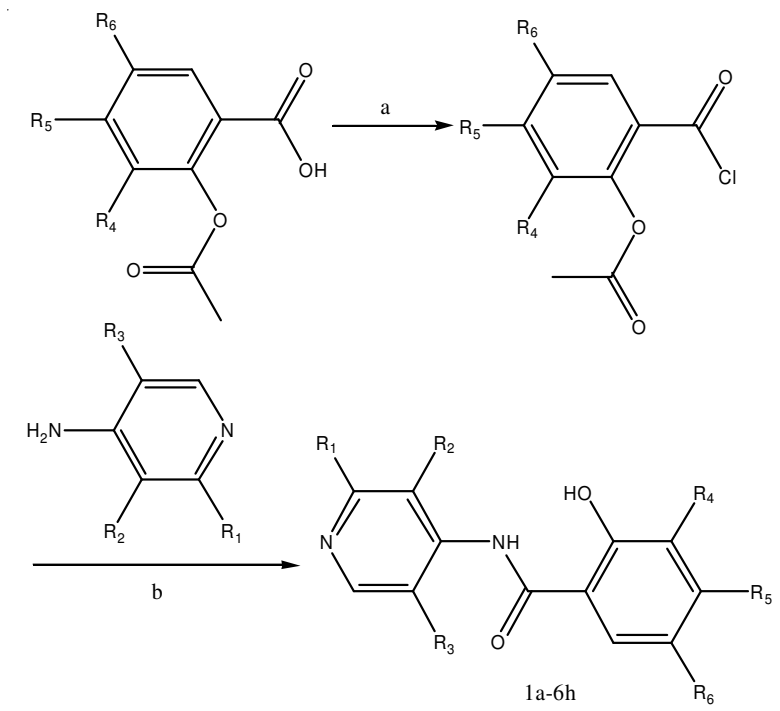

$\mathrm{R}_{1}=\mathrm{H}, \mathrm{Cl}, \mathrm{CH}_{3} ; \mathrm{R}_{2}=\mathrm{H}, \mathrm{Cl}, \mathrm{CF}_{3} ; \mathrm{R}_{3}=\mathrm{R}_{4}=\mathrm{H}, \mathrm{Cl} ; \mathrm{R}_{5}=\mathrm{OCH}_{3} ; \mathrm{R}_{6}=\mathrm{H}, \mathrm{Cl}, \mathrm{Br}, \mathrm{CF}_{3}$ Reagents and conditions: (a) Pyridine, $\mathrm{SOCl}_{2}, 75-100{ }^{\circ} \mathrm{C}$, reflux, 3-5h; (b) $\mathrm{CHCl}_{3}, \mathrm{NEt}_{3}$, r. t., 24-48 h.

Fig. 1. Synthesis of Compounds 1a-6h N-(pyridin-4-yl) salicylamides

\section{EXPERIMENTAL}

Chemistry. melting points (m.p.) were determined on an YRT-3 digital melting point apparatus and were uncorrected. 
Infrared (IR) spectra were measured in $\mathrm{KBr}$ pellets on an EQU NOX-55 instrument. ${ }^{1} \mathrm{H}$ NMR and ${ }^{13} \mathrm{C}$ NMR spectra were recorded in DMSO- $d_{6}$ solution on a Brucker DRX-300M spectrometer operating at $300 \mathrm{MHz}$. Chemical shifts were reported in $\delta$ (ppm) units relative to the internal standard tetramethylsilane (TMS) via the solvent signal (2.49 for ${ }^{1} \mathrm{H}$ or 39.78 for ${ }^{13} \mathrm{C}$ ). EI and FAB mass spectra were obtained on a HP5988 and a HS-ZAB mass spectrometer. Elemental analyses were performed on a CARLOERBA 1106 instrument and the results of elemental analyses for $\mathrm{C}, \mathrm{H}$ and $\mathrm{N}$ were within $\pm 0.4 \%$ of the theoretical values. All chemicals and solvents used were of reagent grade purified and dried by standard methods before use. TLC monitoring all the reaction was performed on precoated silica gel $\mathrm{G}$ plates at 254/365 nm under a UV lamp, with petrol ether/acetone/triethylamine as the mobile phase.

General procedure for the synthesis of compounds of substitued acetylsalicyloyl chlorides: Thionyl chloride $(0.1$ mol) was added dropwise to the mixture of 0.1 mol substituted acetylsalicylic acid and dried pyridine in minute quantities under stirring in the ice bath. The solution was stirred for 0.5 $\mathrm{h}$ at the same temperature, warmed to room temperature and then stirred under $75-90{ }^{\circ} \mathrm{C}$ for further $2.5-5 \mathrm{~h}$. The formed faint yellow liquid was used in the next step without further purification.

General procedure for the synthesis of the compounds of series 1a-6h: To a solution of substituted acetylsalicyloyl chlorides $(0.022 \mathrm{~mol})$ in anhydrous chloroform $(100 \mathrm{~mL})$ was added triethylamine $(0.023 \mathrm{~mol})$ at $4{ }^{\circ} \mathrm{C}$. After the mixture was stirred for 5-15 min and then substituted 4-amino pyridine was added portion wise in the ice bath. After warmed to room temperature, the solution was stirred for $24-48 \mathrm{~h}$ and then quenched by $1 \mathrm{~mL}$ of $1 \mathrm{M}$ hydrochloric acid. The reaction mixture was extracted with $10 \%$ hydrochloric acid (50, 30 and $30 \mathrm{~mL}$ ) and the combined aqueous was basified to $\mathrm{pH} 7$ 9 with cooled saturated sodium bicarbonate solution. The yellow precipitation was filtered producing the crude products. After recrystallized in ethanol, the products were isolated as pure form in more than $85 \%$ yields.

2-Hydroxy-N-(pyridin-4-yl)benzamide (1a): m.p. 233$234{ }^{\circ} \mathrm{C}\left(234-236^{\circ} \mathrm{C}^{[1]}\right)$; m.f. $\mathrm{C}_{12} \mathrm{H}_{10} \mathrm{~N}_{2} \mathrm{O}_{2}$; IR $\left(\mathrm{KBr}, \mathrm{v}_{\max }, \mathrm{cm}^{-1}\right)$ : $(\mathrm{C}=\mathrm{O})$ 1676; ${ }^{1} \mathrm{H}$ NMR: $\delta 10.67$ (bs, $\left.1 \mathrm{H}, N H\right), 8.53-8.45(\mathrm{~m}$, $\left.2 \mathrm{H}, H 2^{\prime}, H 6^{\prime}\right), 7.86(\mathrm{dd}, 1 \mathrm{H}, J=8.10 \mathrm{~Hz}, J=1.80 \mathrm{~Hz}, H 6)$, 7.75-7.73 (m, 2H, H3, H5), 7.47-7.41 (m, 1H, H4), 7.02-6.94 (m, 2H, H3', H5'); ${ }^{13} \mathrm{C}$ NMR: $\delta 166.8,157.5,150.4,145.4$, 133.8, 129.7, 119.3, 118.7, 117.2, 114.3.

N-(3-Chloropyridin-4-yl)-2-hydroxybenzamide (2a): m.p. $187-188{ }^{\circ} \mathrm{C}$; m.f. $\mathrm{C}_{12} \mathrm{H}_{9} \mathrm{~N}_{2} \mathrm{O}_{2} \mathrm{Cl}$; IR $\left(\mathrm{KBr}, v_{\max }, \mathrm{cm}^{-1}\right)$ : $(\mathrm{C}=\mathrm{O}) 1658 ;{ }^{1} \mathrm{H}$ NMR: $\delta 10.52$ (bs, $\left.1 \mathrm{H}, N H\right), 8.69(\mathrm{~s}, 1 \mathrm{H}$, $\left.H 2^{\prime}\right), 8.58\left(\mathrm{~d}, 1 \mathrm{H}, J=8.93 \mathrm{~Hz}, H 6^{\prime}\right), 7.86(\mathrm{dd}, 1 \mathrm{H}, J=7.92 \mathrm{~Hz}$, $J=1.90 \mathrm{~Hz}, H 6), 7.75-7.73(\mathrm{~m}, 2 \mathrm{H}, H 3, H 5), 7.47-7.41(\mathrm{~m}$, $1 \mathrm{H}, H 4), 7.14$ (d, $\left.1 \mathrm{H}, J=8.93 \mathrm{~Hz}, H 5^{\prime}\right) ;{ }^{13} \mathrm{C}$ NMR: $\delta 166.6$, 157.4, 150.2, 143.2, 142.9, 133.6, 129.5, 123.0, 119.4, 118.9, $117.0,115.4$.

2-Hydroxy-N-[3-(trifluoromethyl)pyridin-4-yl]benzamide (3a): m.p. $174-175{ }^{\circ} \mathrm{C}$; m.f. $\mathrm{C}_{13} \mathrm{H}_{9} \mathrm{~F}_{3} \mathrm{~N}_{2} \mathrm{O}_{2}$; IR (KBr, $v_{\max }$, $\left.\mathrm{cm}^{-1}\right)$ : $(\mathrm{C}=\mathrm{O}) 1669 ;{ }^{1} \mathrm{H}$ NMR: $\delta 10.98$ (bs, $\left.1 \mathrm{H}, \mathrm{NH}\right), 8.94-8.85$ (m, 2H, H2', H6'), 7.88 (dd, $1 \mathrm{H}, J=7.97 \mathrm{~Hz}, J=1.84 \mathrm{~Hz}, H 6$ ), 7.77-7.74 (m, 2H, H3, H5), 7.46-7.42 (m, 1H, H4), 8.27 (d, $\left.1 \mathrm{H}, J=8.98 \mathrm{~Hz}, H 5^{\prime}\right) ;{ }^{13} \mathrm{C}$ NMR: d165.9, 157.1, 150.6, 144.1,
$142.3,133.8,128.8,123.8,119.2,118.6,117.5,116.8(J=$ $267 \mathrm{~Hz}), 114.1$.

N-(3-Methylpyridin-4-yl)-2-hydroxybenzamide (4a). m.p. 203-204 ${ }^{\circ} \mathrm{C}$; m.f. $\mathrm{C}_{13} \mathrm{H}_{12} \mathrm{~N}_{2} \mathrm{O}_{2}$; IR $\left(\mathrm{KBr}, \mathrm{v}_{\max }, \mathrm{cm}^{-1}\right)$ : $(\mathrm{C}=\mathrm{O})$ 1683; ${ }^{1} \mathrm{H}$ NMR: $\delta 10.65$ (bs, $\left.1 \mathrm{H}, N H\right), 9.42$ (s, $1 \mathrm{H}, H 2$ '), 8.88 (d, $\left.1 \mathrm{H}, J=7.52 \mathrm{~Hz}, H 6^{\prime}\right), 7.84$ (dd, $1 \mathrm{H}, J=7.20 \mathrm{~Hz}, J=2.00$ $\mathrm{Hz}, H 6), 7.78-7.75$ (m, 2H, H3, H5), 7.49-7.43 (m, 1H, H4), 7.22 (d, $\left.1 \mathrm{H}, J=7.52 \mathrm{~Hz}, H 5^{\prime}\right), 2.41\left(\mathrm{~s}, 3 \mathrm{H},-\mathrm{CH}_{3}\right) ;{ }^{13} \mathrm{C} \mathrm{NMR}$ : S164.9, 157.3, 150.4, 144.7, 141.9, 133.6, 128.3, 123.3, 119.2, 118.7, 117.4, 113.9, 17.7 .

N-(2-Chloropyridin-4-yl)-2-hydroxybenzamide (5a): m.p. 220-221 ${ }^{\circ} \mathrm{C}$; m.f. $\mathrm{C}_{12} \mathrm{H}_{9} \mathrm{~N}_{2} \mathrm{O}_{2} \mathrm{Cl}$; IR $\left(\mathrm{KBr}, \mathrm{v}_{\max }, \mathrm{cm}^{-1}\right)$ : $(\mathrm{C}=\mathrm{O}) 1667 ;{ }^{1} \mathrm{H}$ NMR: $\delta 10.66$ (bs, $\left.1 \mathrm{H}, N H\right), 8.84(\mathrm{~d}, 1 \mathrm{H}, J=$ $\left.8.90 \mathrm{~Hz}, H 6^{\prime}\right), 7.88$ (dd, $\left.1 \mathrm{H}, J=7.92 \mathrm{~Hz}, J=1.73 \mathrm{~Hz}, H 6\right)$, 7.74-7.72 (m, 2H, H3, H5), 7.48-7.43 (m, 1H, H4), 7.26-7.23 $\left(\mathrm{dd}, 2 \mathrm{H}, J=8.90 \mathrm{~Hz}, J=2.60 \mathrm{~Hz}, H 3{ }^{\prime}, H 5^{\prime}\right) ;{ }^{13} \mathrm{C} \mathrm{NMR}: \delta 169.2$, 157.0, 151.1, 144.9, 144.4, 133.8, 129.5, 119.5, 118.7, 117.2, 115.7, 114.3.

N-(3,5-Dichloropyridin-4-yl)-2-hydroxybenzamide (6a): m.p. 230-231 ${ }^{\circ} \mathrm{C}$; m.f. $\mathrm{C}_{12} \mathrm{H}_{8} \mathrm{~N}_{2} \mathrm{O}_{2} \mathrm{Cl}_{2}$; IR (KBr, $v_{\max }, \mathrm{cm}^{-1}$ ): (C=O) 1652; ' ${ }^{1} \mathrm{H}$ NMR: $\delta 10.69$ (bs, $\left.1 \mathrm{H}, N H\right), 8.78$ (s, 2H, H2', H6'), 7.88 (dd, $1 \mathrm{H}, J=7.79 \mathrm{~Hz}, J=1.96 \mathrm{~Hz}, H 6), 7.76-7.73$ (m, 2H, H3, H5), 7.45-7.40 (m, 1H, H4); ${ }^{13} \mathrm{C}$ NMR: $\delta 164.4$, 155.7, 150.4, 141.8, 133.6, 129.3, 119.9, 119.3, 118.7, 117.2.

3,5-Dichloro-2-hydroxy-N-(pyridin-4-yl)benzamide (1b): m.p. 334-336 ${ }^{\circ} \mathrm{C}$; m.f. $\mathrm{C}_{12} \mathrm{H}_{8} \mathrm{~N}_{2} \mathrm{O}_{2} \mathrm{Cl}_{2}$; IR (KBr, $\left.v_{\max }, \mathrm{cm}^{-1}\right)$ : $(\mathrm{C}=\mathrm{O}) 1679 ;{ }^{1} \mathrm{H}$ NMR: $\delta 10.87$ (bs, $\left.1 \mathrm{H}, N H\right), 8.57-8.49$ (m, 2H, H2',H6'), 7.77-7.71 (m, 2H, H3',H5'), 7.76 (d, 1H, J $=2.01 \mathrm{~Hz}, H 6), 7.45(\mathrm{~d}, 1 \mathrm{H}, J=2.01 \mathrm{~Hz}, H 4) ;{ }^{13} \mathrm{C} \mathrm{NMR}: \delta 165.4$, 154.2, 151.3, 146.1, 132.3, 127.9, 126.9, 126.2, 121.5, 114.0.

3,5-Dichloro-N-(3-chloropyridin-4-yl)-2-hydroxybenzamide (2b): m.p. $287-288{ }^{\circ} \mathrm{C}$; m.f. $\mathrm{C}_{12} \mathrm{H}_{7} \mathrm{~N}_{2} \mathrm{O}_{2} \mathrm{Cl}_{3}$; IR $\left(\mathrm{KBr}, \mathrm{v}_{\max }, \mathrm{cm}^{-1}\right):(\mathrm{C}=\mathrm{O}) 1661 ;{ }^{1} \mathrm{H} \mathrm{NMR}: \delta 10.88$ (bs, $\left.1 \mathrm{H}, \mathrm{NH}\right)$, $8.72\left(\mathrm{~s}, 1 \mathrm{H}, H 2^{\prime}\right), 8.54\left(\mathrm{~d}, 1 \mathrm{H}, J=8.94 \mathrm{~Hz}, H 6^{\prime}\right), 7.90$ (d, $1 \mathrm{H}$, $\left.J=8.94 \mathrm{~Hz}, H 5^{\prime}\right), 7.84(\mathrm{~d}, 1 \mathrm{H}, J=2.03, H 6), 7.51(\mathrm{~d}, 1 \mathrm{H}, J$ $=2.03, H 4) ;{ }^{13} \mathrm{C}$ NMR: $\delta 166.8,157.5,150.4,145.4,133.8$, 129.7, 119.3, 118.7, 117.2, 114.3.

3,5-Dichloro-N-(3-(trifluoromethyl)pyridin-4-yl)-2hydroxybenzamide (3b): m.p. $363-265^{\circ} \mathrm{C}$; m.f. $\mathrm{C}_{13} \mathrm{H}_{7} \mathrm{~N}_{2} \mathrm{O}_{2} \mathrm{Cl}_{2} \mathrm{~F}_{3}$; IR $\left(\mathrm{KBr}, \mathrm{v}_{\max }, \mathrm{cm}^{-1}\right)$ : $(\mathrm{C}=\mathrm{O}) 1672 ;{ }^{1} \mathrm{H}$ NMR: $\delta 10.99$ (bs, $1 \mathrm{H}$, $N H), 9.02-8.94$ (m, H2', H6'), 8.19 (d, $\left.1 \mathrm{H}, J=9.02 \mathrm{~Hz}, H 5^{\prime}\right)$, 7.85 (d, $1 \mathrm{H}, J=2.65 \mathrm{~Hz}, H 6), 7.45$ (d, $1 \mathrm{H}, J=2.65 \mathrm{~Hz}, H 4)$; ${ }^{13} \mathrm{C}$ NMR: $\delta 166.8,157.5,150.4,145.4,133.8,129.7,119.3$, $118.7,117.2(\mathrm{~J}=268 \mathrm{~Hz}), 114.0$.

3,5-Dichloro-2-hydroxy-N-(2-methylpyridin-4yl)benzamide (4b): m.p. $356-358^{\circ} \mathrm{C}$; m.f. $\mathrm{C}_{13} \mathrm{H}_{10} \mathrm{~N}_{2} \mathrm{O}_{2} \mathrm{Cl}_{2}$; IR $\left(\mathrm{KBr}, \mathrm{v}_{\max }, \mathrm{cm}^{-1}\right)$ : $(\mathrm{C}=\mathrm{O}) 1686 ;{ }^{1} \mathrm{H}$ NMR: $\delta 10.79(\mathrm{bs}, 1 \mathrm{H}, \mathrm{NH})$, $8.60(\mathrm{~d}, 1 \mathrm{H}, J=7.50 \mathrm{~Hz}, H 6), 7.76(\mathrm{~d}, 1 \mathrm{H}, J=1.99 \mathrm{~Hz}, H 6)$, $7.63\left(\mathrm{~d}, 1 \mathrm{H}, J=2.59 \mathrm{~Hz}, H 3^{\prime}\right), 7.52(\mathrm{dd}, 1 \mathrm{H}, J=7.50 \mathrm{~Hz}, J=$ $\left.2.58 \mathrm{~Hz}, H 5^{\prime}\right), 7.45$ (d, $\left.1 \mathrm{H}, J=2.00 \mathrm{~Hz}, H 4\right), 2.45$ (s, 3H, $\left.-\mathrm{CH}_{3}\right) ;{ }^{13} \mathrm{C}$ NMR: $\delta$ 166.8, 157.5, 150.4, 145.4, 133.8, 129.7, 119.3, 118.7, 117.2, 114.3.

3,5-Dichloro-N-(2-chloropyridin-4-yl)-2-hydroxybenzamide (5b): m.p. 310-311 ${ }^{\circ} \mathrm{C}$; m.f. $\mathrm{C}_{12} \mathrm{H}_{7} \mathrm{~N}_{2} \mathrm{O}_{2} \mathrm{Cl}_{3}$; IR $\left(\mathrm{KBr}, \mathrm{v}_{\max }, \mathrm{cm}^{-1}\right):(\mathrm{C}=\mathrm{O}) 1670 ;{ }^{1} \mathrm{H} \mathrm{NMR}: \delta 10.89(\mathrm{bs}, 1 \mathrm{H}, \mathrm{NH})$, $8.72\left(\mathrm{~d}, 1 \mathrm{H}, J=9.01, H 6^{\prime}\right), 7.77$ (d, $\left.1 \mathrm{H}, J=2.07 \mathrm{~Hz}, H 6\right), 7.45$ (d, $1 \mathrm{H}, J=2.08 \mathrm{~Hz}, H 4), 7.06-6.97$ (m, 2H, H3', H5'); ${ }^{13} \mathrm{C}$ NMR: $\delta 166.8,157.5,150.4,145.4,133.8,129.7,119.3,118.7$, 117.2, 114.3 . 
3,5-Dichloro-N-(3,5-dichloropyridin-4-yl)-2-hydroxybenzamide (6b): m.p. $353-354{ }^{\circ} \mathrm{C}$; m.f. $\mathrm{C}_{12} \mathrm{H}_{6} \mathrm{~N}_{2} \mathrm{O}_{2} \mathrm{Cl}_{4}$; IR ( $\mathrm{KBr}$, $\left.v_{\max }, \mathrm{cm}^{-1}\right)$ : $(\mathrm{C}=\mathrm{O}) 1654 ;{ }^{1} \mathrm{H}$ NMR: $\delta 10.91$ (bs, $\left.1 \mathrm{H}, N H\right), 8.81$ (s, 2H, H2', H6'), 7.81 (d, 1H, $J=2.06 \mathrm{~Hz}, H 6), 7.46$ (d, 1H, $J$ $=2.07 \mathrm{~Hz}, H 4) ;{ }^{13} \mathrm{C}$ NMR: $\delta 166.8,157.5,150.4,145.4,133.8$, 129.7, 119.3, 118.7, 117.2, 114.3.

5-Chloro-2-hydroxy-N-(pyridin-4-yl)benzamide (1c): m.p. 313-315 ${ }^{\circ} \mathrm{C}\left(314-316^{\circ} \mathrm{C}^{[1]}\right)$; m.f. $\mathrm{C}_{12} \mathrm{H}_{9} \mathrm{~N}_{2} \mathrm{O}_{2} \mathrm{Cl}$; IR ( $\mathrm{KBr}$, $\left.\mathrm{V}_{\max }, \mathrm{cm}^{-1}\right):(\mathrm{C}=\mathrm{O}) 1677 ;{ }^{1} \mathrm{H}$ NMR: $\delta 10.83(\mathrm{bs}, 1 \mathrm{H}, N H), 8.52-$ $8.44\left(\mathrm{~m}, 2 \mathrm{H}, H 2^{\prime}, H 6^{\prime}\right), 7.82$ (d, 1H, J = 2.61 Hz, H6), 7.74$7.68\left(\mathrm{~m}, 2 \mathrm{H}, H 3^{\prime}, H 5^{\prime}\right), 7.44(\mathrm{dd}, 1 \mathrm{H}, J=7.88 \mathrm{~Hz}, J=2.60 \mathrm{~Hz}$, H4), 7.01 (d, $1 \mathrm{H}, J=7.88 \mathrm{~Hz}, H 3) ;{ }^{13} \mathrm{C}$ NMR: $\delta 166.0,157.1$, 150.4, 145.4, 133.6, 129.1, 122.3, 120.7, 119.2, 114.1.

5-Chloro-N-(3-chloropyridin-4-yl)-2-hydroxybenzamide (2c): m.p. $355-357^{\circ} \mathrm{C}$; m.f. $\mathrm{C}_{12} \mathrm{H}_{8} \mathrm{~N}_{2} \mathrm{O}_{2} \mathrm{Cl}_{2}$; $\mathrm{IR}$ (KBr, $v_{\max }$, $\left.\mathrm{cm}^{-1}\right)$ : $(\mathrm{C}=\mathrm{O})$ 1659; ${ }^{1} \mathrm{H}$ NMR: $\delta 10.85$ (bs, $\left.1 \mathrm{H}, \mathrm{NH}\right), 8.71$ (s, $\left.1 \mathrm{H}, H 2^{\prime}\right), 8.53\left(\mathrm{~d}, 1 \mathrm{H}, J=9.08 \mathrm{~Hz}, H 6^{\prime}\right), 7.93$ (d, $1 \mathrm{H}, J=9.07$ Hz, H5'), 7.82 (d, 1H, J=1.97 Hz, H6), 7.44 (dd, 1H, $J=7.96$ $\mathrm{Hz}, J=1.97 \mathrm{~Hz}, H 4), 7.01$ (d, $1 \mathrm{H}, J=7.96 \mathrm{~Hz}, H 3) ;{ }^{13} \mathrm{C}$ NMR: $\delta 164.9,156.9,150.2,144.3,143.6,133.3,129.0,122.4,121.7$, $120.9,119.4,115.4$.

5-Chloro-N-(3-(trifluoromethyl)pyridin-4-yl)-2hydroxybenzamide (3c): m.p. $343-342^{\circ} \mathrm{C}$; m.f. $\mathrm{C}_{13} \mathrm{H}_{8} \mathrm{~N}_{2} \mathrm{O}_{2} \mathrm{ClF}_{3}$; IR $\left(\mathrm{KBr}, v_{\max }, \mathrm{cm}^{-1}\right)$ : $(\mathrm{C}=\mathrm{O}) 1672 ;{ }^{1} \mathrm{H}$ NMR: $\delta 10.91(\mathrm{bs}, 1 \mathrm{H}$, $N H)$, 9.00-8.93 (m, 2H, H2', H6'), $8.21(\mathrm{~d}, 1 \mathrm{H}, J=9.01 \mathrm{~Hz}$, $\left.H 5^{\prime}\right), 7.83(\mathrm{~d}, 1 \mathrm{H}, J=2.72 \mathrm{~Hz}, H 6), 7.46(\mathrm{dd}, 1 \mathrm{H}, J=7.93 \mathrm{~Hz}$, $J=2.72 \mathrm{~Hz}, H 4), 7.01(\mathrm{~d}, 1 \mathrm{H}, J=7.92 \mathrm{~Hz}, H 3) ;{ }^{13} \mathrm{C}$ NMR: $\delta$ $165.1,157.0,150.5,146.3,143.3,133.4,129.2,122.3,120.8$, $120.1,119.3,117.4(J=271 \mathrm{~Hz}), 113.9$.

5-Chloro-2-hydroxy-N-(2-methylpyridin-4-yl)benzamide (4c): m.p. $338-339^{\circ} \mathrm{C}$; m.f. $\mathrm{C}_{13} \mathrm{H}_{11} \mathrm{~N}_{2} \mathrm{O}_{2} \mathrm{Cl}$; IR (KBr, $v_{\max }$, $\left.\mathrm{cm}^{-1}\right)$ : $(\mathrm{C}=\mathrm{O}) 1684 ;{ }^{1} \mathrm{H}$ NMR: $\delta 10.76(\mathrm{bs}, 1 \mathrm{H}, \mathrm{NH}), 8.59(\mathrm{~d}$, $\left.1 \mathrm{H}, J=7.58 \mathrm{~Hz}, H 6^{\prime}\right), 7.79$ (d, $\left.1 \mathrm{H}, J=2.31 \mathrm{~Hz}, H 6\right), 7.61-$ 7.49 (m, 2H, H3', H5'), 7.42 (dd, $1 \mathrm{H}, J=7.96 \mathrm{~Hz}, J=2.31 \mathrm{~Hz}$, H4), 6.99 (d, $1 \mathrm{H}, J=7.96 \mathrm{~Hz}, H 3), 2.50\left(\mathrm{~s}, 3 \mathrm{H},-\mathrm{CH}_{3}\right) ;{ }^{13} \mathrm{C}$ NMR: $\delta 165.2,157.3,157.0,150.6,144.8,133.5,128.9,122.1$, 121.0, 119.0, 114.5, 112.6, 24.1.

5-Chloro-N-(2-chloropyridin-4-yl)-2-hydroxybenzamide (5c): m.p. $354-356{ }^{\circ} \mathrm{C}$; m.f. $\mathrm{C}_{12} \mathrm{H}_{8} \mathrm{~N}_{2} \mathrm{O}_{2} \mathrm{Cl}_{2}$; $\mathrm{IR}$ (KBr, $v_{\max }$, $\left.\mathrm{cm}^{-1}\right)$ : $(\mathrm{C}=\mathrm{O}) 1668 ;{ }^{1} \mathrm{H}$ NMR: $\delta 10.86(\mathrm{bs}, 1 \mathrm{H}, \mathrm{NH}), 8.70(\mathrm{~d}$, $\left.1 \mathrm{H}, J=9.04, H 6^{\prime}\right), 8.06-7.97$ (m, 2H, H2', H6'), 7.81 (d, 1H, $J$ $=2.63 \mathrm{~Hz}, H 6), 7.74-7.68\left(\mathrm{~m}, 2 \mathrm{H}, H 3^{\prime}, H 5^{\prime}\right), 7.43(\mathrm{dd}, 1 \mathrm{H}, J=$ $7.72 \mathrm{~Hz}, J=2.63 \mathrm{~Hz}, H 4), 7.01(\mathrm{~d}, 1 \mathrm{H}, J=7.71 \mathrm{~Hz}, H 3) ;{ }^{13} \mathrm{C}$ NMR: $\delta 166.3,158.5,157.4,145.7,144.6,133.4,129.3,122.2$, $120.7,119.2,115.5,114.4$.

5-Chloro-N-(3,5-dichloropyridin-4-yl)-2-hydroxybenzamide (6c): m.p. $391-393{ }^{\circ} \mathrm{C}$; m.f. $\mathrm{C}_{12} \mathrm{H}_{7} \mathrm{~N}_{2} \mathrm{O}_{2} \mathrm{Cl}_{3}$; IR $\left(\mathrm{KBr}, v_{\max }, \mathrm{cm}^{-1}\right):(\mathrm{C}=\mathrm{O}) 1653 ;{ }^{1} \mathrm{H}$ NMR: $\delta 10.89(\mathrm{bs}, 1 \mathrm{H}, \mathrm{NH})$, $8.81\left(\mathrm{~s}, 2 \mathrm{H}, H 2^{\prime}, H 6^{\prime}\right), 7.82(\mathrm{~d}, 1 \mathrm{H}, J=2.58 \mathrm{~Hz}, H 6), 7.43$ (dd, $1 \mathrm{H}, J=8.09 \mathrm{~Hz}, J=2.58 \mathrm{~Hz}, H 4), 7.01(\mathrm{~d}, 1 \mathrm{H}, J=8.08 \mathrm{~Hz}$, H3); ${ }^{13} \mathrm{C}$ NMR: $\delta 166.7,157.5,149.1,142.6,133.6,129.1$, $122.3,120.7,120.2,118.9$.

3-Chloro-2-hydroxy-N-(pyridin-4-yl)benzamide (1d): m.p. 296-297 ${ }^{\circ} \mathrm{C}$; m.f. $\mathrm{C}_{12} \mathrm{H}_{9} \mathrm{~N}_{2} \mathrm{O}_{2} \mathrm{Cl}$; IR $\left(\mathrm{KBr}, \mathrm{v}_{\max }, \mathrm{cm}^{-1}\right)$ : $(\mathrm{C}=\mathrm{O})$ 1678; 'H NMR: $\delta 10.84$ (bs, $1 \mathrm{H}, \mathrm{NH}$ ), 8.53-8.45 (m, 2H, H2', $\left.H 6^{\prime}\right), 7.69(\mathrm{dd}, 1 \mathrm{H}, J=7.61 \mathrm{~Hz}, J=2.58 \mathrm{~Hz}, H 6), 7.76-7.70(\mathrm{~m}$, 2H, H3', H5'), 7.43 (dd, $1 \mathrm{H}, J=7.89 \mathrm{~Hz}, J=2.58 \mathrm{~Hz}, H 4), 7.05$ (t, $1 \mathrm{H}, J=7.89 \mathrm{~Hz}, J=7.61 \mathrm{~Hz}, H 5) ;{ }^{13} \mathrm{C}$ NMR: $\delta 166.4,157.7$, $150.3,145.2$, 133.1, 127.0, 121.3, 120.9, 119.0, 113.8 .
3-Chloro-N-(3-chloropyridin-4-yl)-2-hydroxybenzamide (2d): m.p. $271-273{ }^{\circ} \mathrm{C}$; m.f. $\mathrm{C}_{12} \mathrm{H}_{8} \mathrm{~N}_{2} \mathrm{O}_{2} \mathrm{Cl}_{2}$; IR (KBr, $v_{\max }$, $\left.\mathrm{cm}^{-1}\right)$ : $(\mathrm{C}=\mathrm{O})$ 1650; ${ }^{1} \mathrm{H}$ NMR: $\delta 10.86$ (bs, $\left.1 \mathrm{H}, \mathrm{NH}\right), 8.72$ (s, $\left.1 \mathrm{H}, H 2^{\prime}\right), 8.58$ (d, $\left.1 \mathrm{H}, J=9.01 \mathrm{~Hz}, H 6^{\prime}\right), 7.91$ (d, $1 \mathrm{H}, J=9.00$ $\left.\mathrm{Hz}, H 5^{\prime}\right), 7.67(\mathrm{dd}, 1 \mathrm{H}, J=7.58 \mathrm{~Hz}, J=2.53 \mathrm{~Hz}, H 6), 7.41$ (dd, $1 \mathrm{H}, J=7.82 \mathrm{~Hz}, J=2.53 \mathrm{~Hz}, H 4), 7.10$ (t, $1 \mathrm{H}, J=7.81$ $\mathrm{Hz}, J=7.56 \mathrm{~Hz}, H 5) ;{ }^{13} \mathrm{C}$ NMR: $\delta 166.6,157.5,150.1,144.1$, 143.8, 133.3, 126.9, 121.1, 120.8, 119.1, 118.4, 114.8 .

3-Chloro-N-[3-(trifluoromethyl)pyridin-4-yl]-2hydroxybenzamide (3d): m.p. $256-257^{\circ} \mathrm{C}$; m.f. $\mathrm{C}_{13} \mathrm{H}_{8} \mathrm{~N}_{2} \mathrm{O}_{2} \mathrm{ClF}_{3}$; IR (KBr, $\left.v_{\max }, \mathrm{cm}^{-1}\right)$ : $(\mathrm{C}=\mathrm{O}) 1669 ;{ }^{1} \mathrm{H}$ NMR: $\delta 10.90(\mathrm{bs}, 1 \mathrm{H}$, $N H)$, 8.97-8.92 (m, 2H, H2',H6'), 8.23 (d, $1 \mathrm{H}, J=9.09 \mathrm{~Hz}$, $\left.H 5^{\prime}\right), 7.82(\mathrm{dd}, 1 \mathrm{H}, J=7.90 \mathrm{~Hz}, J=2.69 \mathrm{~Hz}, \mathrm{H} 6), 7.45$ (dd, $1 \mathrm{H}, J=7.97 \mathrm{~Hz}, J=2.69 \mathrm{~Hz}, H 4), 7.11(\mathrm{t}, 1 \mathrm{H}, J=7.96 \mathrm{~Hz}, J$ $=7.90 \mathrm{~Hz}, \mathrm{H} 5) ;{ }^{13} \mathrm{C}$ NMR: $\delta 166.6,157.6,150.5,146.0,143.2$, 133.2, 127.1, 121.5, 120.6, 119.6, 118.9, $117.7(J=266 \mathrm{~Hz})$, 113.4 .

3-Chloro-2-hydroxy-N-(2-methylpyridin-4-yl)benzamide (4d ): m.p. 268-270 ${ }^{\circ} \mathrm{C}$; m.f. $\mathrm{C}_{13} \mathrm{H}_{11} \mathrm{~N}_{2} \mathrm{O}_{2} \mathrm{Cl}$; IR ( $\mathrm{KBr}$, $\left.v_{\max }, \mathrm{cm}^{-1}\right):(\mathrm{C}=\mathrm{O}) 1685 ;{ }^{1} \mathrm{H}$ NMR: $\delta 10.74(\mathrm{bs}, 1 \mathrm{H}, N H), 8.60$ (d, $\left.1 \mathrm{H}, J=7.52 \mathrm{~Hz}, H 6^{\prime}\right), 7.63-7.52$ (m, 2H, H3', H5'), 7.66 $(\mathrm{dd}, 1 \mathrm{H}, J=7.53 \mathrm{~Hz}, J=2.52 \mathrm{~Hz}, H 6), 7.39(\mathrm{dd}, 1 \mathrm{H}, J=7.86$ $\mathrm{Hz}, J=2.52 \mathrm{~Hz}, H 4), 7.02(\mathrm{t}, 1 \mathrm{H}, J=7.85 \mathrm{~Hz}, J=7.53 \mathrm{~Hz}$, H5), 2.49 (s, 3H, $-\mathrm{CH}_{3}$ ); ${ }^{13} \mathrm{C}$ NMR: $\delta 165.7,158.3,157.8,150.4$, 144.5, 133.0, 127.1, 121.4, 120.7, 119.2, 114.2, 112.3, 23.9.

3-Chloro-N-(2-chloropyridin-4-yl)-2-hydroxybenzamide (5d). m.p. 243-246 ${ }^{\circ} \mathrm{C}$; m.f. $\mathrm{C}_{12} \mathrm{H}_{8} \mathrm{~N}_{2} \mathrm{O}_{2} \mathrm{Cl}_{2}$; IR (KBr, $v_{\max }$, $\left.\mathrm{cm}^{-1}\right)$ : $(\mathrm{C}=\mathrm{O}) 1669 ;{ }^{1} \mathrm{H}$ NMR: $\delta 10.87$ (bs, $\left.1 \mathrm{H}, N H\right), 8.72(\mathrm{~d}$, $\left.1 \mathrm{H}, J=9.07, H 6^{\prime}\right), 8.06-7.97\left(\mathrm{~m}, 2 \mathrm{H}, H 3^{\prime}, H 5^{\prime}\right), 7.68(\mathrm{dd}, 1 \mathrm{H}$, $J=7.59 \mathrm{~Hz}, J=2.53 \mathrm{~Hz}, H 6), 7.42(\mathrm{dd}, 1 \mathrm{H}, J=7.86 \mathrm{~Hz}, J=$ $2.53 \mathrm{~Hz}, H 4), 7.05$ (t, $1 \mathrm{H}, J=7.87 \mathrm{~Hz}, J=7.59 \mathrm{~Hz}, H 5) ;{ }^{13} \mathrm{C}$ NMR: $\delta 166.6,158.1,157.3,145.5,144.0,133.0,127.2,121.3$, 120.7, 118.8, 115.2, 114.1.

3-Chloro-N-(3,5-dichloropyridin-4-yl)-2-hydroxybenzamide (6d): m.p. $287-288{ }^{\circ} \mathrm{C}$; m.f. $\mathrm{C}_{12} \mathrm{H}_{7} \mathrm{~N}_{2} \mathrm{O}_{2} \mathrm{Cl}_{3}$; IR $\left(\mathrm{KBr}, \mathrm{v}_{\max }, \mathrm{cm}^{-1}\right):(\mathrm{C}=\mathrm{O}) 1654 ;{ }^{1} \mathrm{H} \mathrm{NMR}: \delta 10.90(\mathrm{bs}, 1 \mathrm{H}, \mathrm{NH})$, $8.81\left(\mathrm{~s}, 2 \mathrm{H}, H 2^{\prime}, H 6^{\prime}\right), 7.70(\mathrm{dd}, 1 \mathrm{H}, J=7.71 \mathrm{~Hz}, J=2.62 \mathrm{~Hz}$, H6), $7.42(\mathrm{dd}, 1 \mathrm{H}, J=7.93 \mathrm{~Hz}, J=2.62 \mathrm{~Hz}, H 4), 7.05(\mathrm{t}, 1 \mathrm{H}$, $J=7.93 \mathrm{~Hz}, J=7.71 \mathrm{~Hz}, H 5) ;{ }^{13} \mathrm{C} \mathrm{NMR}: \delta 165.5,157.5,149.5$, 142.4, 129.7, 126.8, 121.0, 120.6, 119.4, 118.5.

5-Bromo-2-hydroxy-N-(pyridin-4-yl)benzamide (1e): m.p. 302-303 ${ }^{\circ} \mathrm{C}\left(304-305^{\circ} \mathrm{C}^{[1]}\right)$; m.f. $\mathrm{C}_{12} \mathrm{H}_{9} \mathrm{~N}_{2} \mathrm{O}_{2} \mathrm{Br}$; IR (KBr, $\left.\mathrm{V}_{\max }, \mathrm{cm}^{-1}\right):(\mathrm{C}=\mathrm{O}) 1678 ;{ }^{1} \mathrm{H}$ NMR: $\delta 10.84(\mathrm{bs}, 1 \mathrm{H}, \mathrm{NH}), 8.50-$ 8.44 (m, H2', H6'), 7.93 (d, 1H, $J=2.46 \mathrm{~Hz}, H 6), 7.72-7.68$ (m, 2H, H3', H5'), 7.55 (dd, $1 \mathrm{H}, J=8.78 \mathrm{~Hz}, J=2.47 \mathrm{~Hz}, H 4)$, $6.97(\mathrm{~d}, 1 \mathrm{H}, J=8.78 \mathrm{~Hz}, H 3) ;{ }^{13} \mathrm{C} \mathrm{NMR}: \delta 165.6,157.1,150.4$, $145.5,136.3,131.7,121.3,119.7,114.2,110.2$.

5-Bromo-N-(3-chloropyridin-4-yl)-2-hydroxybenzamide (2e): m.p. 343-345 ${ }^{\circ} \mathrm{C}$; m.f. $\mathrm{C}_{12} \mathrm{H}_{8} \mathrm{~N}_{2} \mathrm{O}_{2} \mathrm{BrCl}$; IR $\left(\mathrm{KBr}, \mathrm{v}_{\max }, \mathrm{cm}^{-1}\right)$ : $(\mathrm{C}=\mathrm{O}) 1659 ;{ }^{1} \mathrm{H}$ NMR: $\delta 10.85$ (bs, $\left.1 \mathrm{H}, \mathrm{NH}\right)$, 8.71 (s, 1H, H2'), 8.54 (d, 1H, $\left.J=8.97 \mathrm{~Hz}, H 6^{\prime}\right), 7.94$ (d, overlapped, $1 \mathrm{H}, J=2.46 \mathrm{~Hz}, H 6), 7.92$ (d, overlapped, $1 \mathrm{H}, J$ $\left.=8.96 \mathrm{~Hz}, H 5^{\prime}\right), 7.55(\mathrm{dd}, 1 \mathrm{H}, J=8.79 \mathrm{~Hz}, J=2.47 \mathrm{~Hz}, H 4)$, $6.96(\mathrm{~d}, 1 \mathrm{H}, J=8.79 \mathrm{~Hz}, H 3) ;{ }^{13} \mathrm{C} \mathrm{NMR}: \delta 165.2,157.0,150.2$, 144.5, 144.1, 136.2, 131.6, 121.1, 119.6, 118.6, 114.4, 111.5.

5-Bromo-N-(3-[trifluoromethyl)pyridin-4-yl]-2-hydroxybenzamide (3e): m.p. $331-333{ }^{\circ} \mathrm{C}$; m.f. $\mathrm{C}_{13} \mathrm{H}_{8} \mathrm{~N}_{2} \mathrm{O}_{2} \mathrm{BrF}_{3}$; IR $\left(\mathrm{KBr}, \mathrm{v}_{\max }, \mathrm{cm}^{-1}\right)$ : (C=O) 1670; ${ }^{1} \mathrm{H}$ NMR: $\delta 10.89$ (bs, $\left.1 \mathrm{H}, \mathrm{NH}\right)$, 8.89-8.85 (m, H2', H6'), 7.94 (d, 1H, J = 2.48 Hz, H6), 8.22(d, 
$\left.1 \mathrm{H}, J=9.02 \mathrm{~Hz}, H 5^{\prime}\right), 7.55(\mathrm{dd}, 1 \mathrm{H}, J=8.81 \mathrm{~Hz}, J=2.48 \mathrm{~Hz}$, H4), 6.97 (d, $1 \mathrm{H}, J=8.80 \mathrm{~Hz}, H 3) ;{ }^{13} \mathrm{C}$ NMR: $\delta 165.8,157.2$, 150.6, 146.3, 143.5, 136.2, 131.5, 121.4, 119.9, 119.4, 115.1, $114.9(\mathrm{~J}=266 \mathrm{~Hz}), 110$.

5-Bromo-2-hydroxy-N-(2-methylpyridin-4-yl)-benzamide (4e): m.p. 299-301 ${ }^{\circ} \mathrm{C}$; m.f. $\mathrm{C}_{13} \mathrm{H}_{11} \mathrm{~N}_{2} \mathrm{O}_{2} \mathrm{Br}$; IR (KBr, $\mathrm{v}_{\max }$, $\left.\mathrm{cm}^{-1}\right)$ : $(\mathrm{C}=\mathrm{O}) 1684 ;{ }^{1} \mathrm{H}$ NMR: $\delta 10.71(\mathrm{bs}, 1 \mathrm{H}, N H), 8.59(\mathrm{~d}$, $\left.1 \mathrm{H}, J=7.56 \mathrm{~Hz}, H 6^{\prime}\right), 7.62-7.54\left(\mathrm{~m}, 2 \mathrm{H}, H 3^{\prime}, H 5^{\prime}\right), 7.91$ (d, $1 \mathrm{H}, J=1.96 \mathrm{~Hz}, H 6), 7.53(\mathrm{dd}, 1 \mathrm{H}, J=8.93 \mathrm{~Hz}, J=1.96 \mathrm{~Hz}$, H4), $6.94(\mathrm{~d}, 1 \mathrm{H}, J=8.93 \mathrm{~Hz}, H 3), 2.45\left(\mathrm{~s}, 3 \mathrm{H},-\mathrm{CH}_{3}\right) ;{ }^{13} \mathrm{C}$ NMR: $\delta 165.9,158.6,157.0,150.6,146.9,136.4,131.6,121.0$, 119.9, 114.4, 110.6, 108.7, 23.9.

5-Bromo-N-(2-chloropyridin-4-yl)-2-hydroxybenzamide (5e): m.p. 274- $275{ }^{\circ} \mathrm{C}$; m.f. $\mathrm{C}_{12} \mathrm{H}_{8} \mathrm{~N}_{2} \mathrm{O}_{2} \mathrm{BrCl}$; IR ( $\mathrm{KBr}$, $\left.v_{\max }, \mathrm{cm}^{-1}\right):(\mathrm{C}=\mathrm{O}) 1666 ;{ }^{1} \mathrm{H}$ NMR: $\delta 10.83$ (bs, $\left.1 \mathrm{H}, N H\right), 8.70$ (d, $\left.1 \mathrm{H}, J=9.04, H 6^{\prime}\right), 8.05-7.98$ (m, 2H, H3', H5'), 7.94 (d, $1 \mathrm{H}, J=2.52 \mathrm{~Hz}, H 6), 7.56(\mathrm{dd}, 1 \mathrm{H}, J=8.74 \mathrm{~Hz}, J=2.51 \mathrm{~Hz}$, H4), 6.96 (d, $1 \mathrm{H}, J=8.75 \mathrm{~Hz}, H 3) ;{ }^{13} \mathrm{C}$ NMR: $\delta 165.6,156.7$, 156.4, 145.8, 144.3, 136.5, 131.4, 121.6, 119.5, 114.1, 111,6, 110.5 .

5-Bromo-N-(3, 5-dichloropyridin-4-yl)-2-hydroxybenzamide (6e): m.p. $317-319{ }^{\circ} \mathrm{C}$; m.f. $\mathrm{C}_{12} \mathrm{H}_{7} \mathrm{~N}_{2} \mathrm{O}_{2} \mathrm{BrCl}_{2}$; IR $\left(\mathrm{KBr}, \mathrm{v}_{\max }, \mathrm{cm}^{-1}\right):(\mathrm{C}=\mathrm{O}) 1653 ;{ }^{1} \mathrm{H} \mathrm{NMR}: \delta 10.88(\mathrm{bs}, 1 \mathrm{H}, \mathrm{NH})$, 8.80-8.74 (m, H2', H6'), 7.94 (d, $1 \mathrm{H}, J=2.52 \mathrm{~Hz}, H 6), 7.56$ $(\mathrm{dd}, 1 \mathrm{H}, J=8.84 \mathrm{~Hz}, J=2.52 \mathrm{~Hz}, H 4), 6.96(\mathrm{~d}, 1 \mathrm{H}, J=8.84$ $\mathrm{Hz}, H 3) ;{ }^{13} \mathrm{C}$ NMR: $\delta 166.8,157.5,150.4,145.4,133.8,129.7$, 119.3, 118.7, 117.2, 114.3.

5-(Trifluoromethyl)-2-hydroxy-N-(pyridin-4-yl)benzamide (1f): m.p. $261-263{ }^{\circ} \mathrm{C}$; m.f. $\mathrm{C}_{13} \mathrm{H}_{9} \mathrm{~N}_{2} \mathrm{O}_{2} \mathrm{~F}_{3}$; IR (KBr, $v_{\max }$, $\left.\mathrm{cm}^{-1}\right)$ : $(\mathrm{C}=\mathrm{O}) 1732 ;{ }^{1} \mathrm{H}$ NMR: $\delta 11.57$ (bs, $\left.1 \mathrm{H}, \mathrm{NH}\right), 8.55-8.48$ $\left(\mathrm{m}, 2 \mathrm{H}, H 2^{\prime}, H 6^{\prime}\right), 8.28(\mathrm{~d}, 1 \mathrm{H}, J=2.30 \mathrm{~Hz}, H 6), 7.81(\mathrm{dd}, 1 \mathrm{H}$, $J=8.31 \mathrm{~Hz}, J=2.30 \mathrm{~Hz}, H 4), 7.21(\mathrm{~d}, 1 \mathrm{H}, J=8.30 \mathrm{~Hz}, H 3)$, 7.04-6.97 (m, 2H, H3', H5'); ${ }^{13} \mathrm{C}$ NMR: $\delta$ 165.3, 161.4, 151.2, $145.7,130.1,124.9,121.6(J=270 \mathrm{~Hz}), 120.2$, 119.4, 117.8, 110.3 .

N-(3-Chloropyridin-4-yl)-5-(trifluoromethyl)-2hydroxybenzamide (2f): m.p. $232-234{ }^{\circ} \mathrm{C}$; m.f. $\mathrm{C}_{13} \mathrm{H}_{8} \mathrm{~N}_{2} \mathrm{O}_{2} \mathrm{ClF}_{3}$; IR $\left(\mathrm{KBr}, v_{\max }, \mathrm{cm}^{-1}\right):(\mathrm{C}=\mathrm{O}) 1714 ;{ }^{1} \mathrm{H}$ NMR: $\delta 11.56(\mathrm{bs}, 1 \mathrm{H}$, $N H$ ), 8.72 (s, 1H, H2'), 8.61 (d, 1H, J = 9.07 Hz, H6'), 8.29 (d, $1 \mathrm{H}, J=2.32 \mathrm{~Hz}, H 6), 7.91\left(\mathrm{~d}, 1 \mathrm{H}, J=9.06 \mathrm{~Hz}, H 5^{\prime}\right), 7.81$ (dd, $1 \mathrm{H}, J=8.33 \mathrm{~Hz}, J=2.33 \mathrm{~Hz}, H 4), 7.22(\mathrm{~d}, 1 \mathrm{H}, J=8.32 \mathrm{~Hz}$, H3); ${ }^{13} \mathrm{C}$ NMR: $\delta 165.5,161.3,151.4,144.5,143.6,130.0$, $124.8,121.5(\mathrm{~J}=270 \mathrm{~Hz}), 120.3,119.5,119.1$, 117.7, 111.6.

5-(Trifluoromethyl)-N-(3-(trifluoromethyl)pyridin-4yl)-2-hydroxybenzamide (3f): m.p. 219-221 ${ }^{\circ} \mathrm{C}$; m.f. $\mathrm{C}_{14} \mathrm{H}_{8} \mathrm{~N}_{2} \mathrm{O}_{2} \mathrm{~F}_{6}$; IR $\left(\mathrm{KBr}, v_{\max }, \mathrm{cm}^{-1}\right)$ : $(\mathrm{C}=\mathrm{O}) 1725 ;{ }^{1} \mathrm{H}$ NMR: $\delta 11.62$ (bs, $1 \mathrm{H}, N H), 8.91-8.87\left(\mathrm{~m}, 2 \mathrm{H}, H 2^{\prime}, H 6^{\prime}\right), 8.29$ (d, $1 \mathrm{H}, J=2.32 \mathrm{~Hz}, H 6), 8.22\left(\mathrm{~d}, 1 \mathrm{H}, J=9.05 \mathrm{~Hz}, H 5^{\prime}\right), 7.81(\mathrm{dd}$, $1 \mathrm{H}, J=8.28 \mathrm{~Hz}, J=2.33 \mathrm{~Hz}, H 4), 7.21(\mathrm{~d}, 1 \mathrm{H}, J=8.28 \mathrm{~Hz}$, H3); ${ }^{13} \mathrm{C}$ NMR: $\delta 166.1,161.6,151.4,146.5,143.5,130.2$, $124.8,121.5(J=270 \mathrm{~Hz}), 120.3,119.2,118.7,117.8,117.3$ $(J=266 \mathrm{~Hz}), 110.1$.

5-(Trifluoromethyl)-2-hydroxy-N-(2-methylpyridin-4yl)benzamide (4f): m.p. $255-256{ }^{\circ} \mathrm{C}$; m.f. $\mathrm{C}_{14} \mathrm{H}_{11} \mathrm{~N}_{2} \mathrm{O}_{2} \mathrm{~F}_{3}$; IR $\left(\mathrm{KBr}, v_{\max }, \mathrm{cm}^{-1}\right)$ : $(\mathrm{C}=\mathrm{O}) 1739 ;{ }^{1} \mathrm{H}$ NMR: $\delta 11.44$ (bs, $\left.1 \mathrm{H}, \mathrm{NH}\right)$, $8.60\left(\mathrm{~d}, 1 \mathrm{H}, J=7.58 \mathrm{~Hz}, H 6^{\prime}\right), 8.25(\mathrm{~d}, 1 \mathrm{H}, J=1.83 \mathrm{~Hz}, H 6)$, $7.62-7.53\left(\mathrm{~m}, 2 \mathrm{H}, H 3^{\prime}, H 5^{\prime}\right), 7.77(\mathrm{dd}, 1 \mathrm{H}, J=8.16 \mathrm{~Hz}, J=$ $1.83 \mathrm{~Hz}, H 4), 7.14$ (d, $1 \mathrm{H}, J=8.16 \mathrm{~Hz}, H 3), 2.47$ (s, 3H, $\left.-\mathrm{CH}_{3}\right) ;{ }^{13} \mathrm{C}$ NMR: $\delta 165.5,161.3,154.6,151.4,145.0,130.2$,
$124.8,121.4(J=270 \mathrm{~Hz}), 120.2,119.3,117.8,110.7,108.8$, 23.8 .

N-(2-Chloropyridin-4-yl)-5-(trifluoromethyl)-2hydroxybenzamide (5f): m.p. $233-235^{\circ} \mathrm{C}$; m.f. $\mathrm{C}_{13} \mathrm{H}_{8} \mathrm{~N}_{2} \mathrm{O}_{2} \mathrm{ClF}_{3}$; IR (KBr, $\left.v_{\max }, \mathrm{cm}^{-1}\right):(\mathrm{C}=\mathrm{O}) 1723 ;{ }^{1} \mathrm{H}$ NMR: $\delta 11.56$ (bs, $1 \mathrm{H}$, $N H), 8.72\left(\mathrm{~d}, 1 \mathrm{H}, J=9.06, H 6^{\prime}\right), 8.05-8.01$ (m, 2H, H3', H5'), $8.29(\mathrm{~d}, 1 \mathrm{H}, J=2.36 \mathrm{~Hz}, H 6), 7.82(\mathrm{dd}, 1 \mathrm{H}, J=8.35 \mathrm{~Hz}, J=$ $2.37 \mathrm{~Hz}, H 4), 7.23$ (d, $1 \mathrm{H}, J=8.35 \mathrm{~Hz}, H 3) ;{ }^{13} \mathrm{C}$ NMR: $\delta 166.3$, 161.7 154.5, 146.0, 145.6, 130.2, 125.1, $121.5(J=270 \mathrm{~Hz})$, 120.2, 119.3, 117.6, 111.7, 110.6.

N-(3,5-Dichloropyridin-4-yl)-5-(trifluoromethyl)-2hydroxybenzamide (6f): m.p. $273-275^{\circ} \mathrm{C}$; m.f. $\mathrm{C}_{13} \mathrm{H}_{7} \mathrm{~N}_{2} \mathrm{O}_{2} \mathrm{Cl}_{2} \mathrm{~F}_{3}$; IR $\left(\mathrm{KBr}, \mathrm{v}_{\max }, \mathrm{cm}^{-1}\right)$ : $(\mathrm{C}=\mathrm{O}) 1708 ;{ }^{1} \mathrm{H}$ NMR: $\delta 11.61$ (bs, $1 \mathrm{H}$, $N H)$, 8.81-8.76 (m, H2',H6'), 8.29 (d, $1 \mathrm{H}, J=2.37 \mathrm{~Hz}, H 6$ ), $7.82(\mathrm{dd}, 1 \mathrm{H}, J=8.38 \mathrm{~Hz}, J=2.38 \mathrm{~Hz}, H 4), 7.20(\mathrm{~d}, 1 \mathrm{H}, J=$ $8.38 \mathrm{~Hz}, H 3) ;{ }^{13} \mathrm{C}$ NMR: $\delta 165.5,161.3,150.8,142.9,130.0$, 124.7, $121.8(\mathrm{~J}=270 \mathrm{~Hz}), 120.4,119.2,118.7,117.7$.

5-Chloro-2-hydroxy-4-methoxy-N-(pyridin-4-yl)benzamide (1g): m.p. 333-334 ${ }^{\circ} \mathrm{C}$; m.f. $\mathrm{C}_{13} \mathrm{H}_{11} \mathrm{~N}_{2} \mathrm{O}_{3} \mathrm{Cl}$; IR $\left(\mathrm{KBr}, \mathrm{v}_{\max }, \mathrm{cm}^{-1}\right)$ : $(\mathrm{C}=\mathrm{O}) 1678 ;{ }^{1} \mathrm{H} \mathrm{NMR}: \delta 10.80$ (bs, $\left.1 \mathrm{H}, \mathrm{NH}\right)$, 8.51-8.45 (m, 2H, H2', H6'), 7.73-7.69 (m, 2H, H3', H5'), 7.71 (s, 1H, H6), 6.52 (s, 1H, H3), $3.76\left(\mathrm{~s}, 3 \mathrm{H},-\mathrm{CH}_{3}\right) ;{ }^{13} \mathrm{C} \mathrm{NMR:} \delta$ 166.7, 160.2, 156.6, 150.3, 145.0, 130.9, 114.6, 114.1, 113.7, 103.0, 55.5.

5-Chloro-N-(3-chloropyridin-4-yl)-2-hydroxy-4methoxybenzamide (2g): m.p. $365-367^{\circ} \mathrm{C}$; m.f. $\mathrm{C}_{13} \mathrm{H}_{10} \mathrm{~N}_{2} \mathrm{O}_{3} \mathrm{Cl}_{2}$; IR $\left(\mathrm{KBr}, v_{\max }, \mathrm{cm}^{-1}\right)$ : $(\mathrm{C}=\mathrm{O}) 1660 ;{ }^{1} \mathrm{H}$ NMR: $\delta 10.81$ (bs, $1 \mathrm{H}$, $N H$ ), 8.69 (s, 1H, H2'), 8.63 (d, 1H, J = 9.55 Hz, H6'), 7.94 (d, $\left.1 \mathrm{H}, J=9.56 \mathrm{~Hz}, H 5^{\prime}\right), 7.75$ (s, 1H, H6), 6.54 (s, 1H, H3), 3.74 (s, $\left.3 \mathrm{H},-\mathrm{CH}_{3}\right) ;{ }^{13} \mathrm{C}$ NMR: $\delta 166.7,160.1,156.7,150.2,144.0$, 143.6, 130.6, 122.5, 114.6, 114.3, 114.0, 103.0, 55.6.

5-Chloro-N-(3-(trifluoromethyl)pyridin-4-yl)-2hydroxy-4-methoxybenzamide (3g): m.p. 349-352 ${ }^{\circ} \mathrm{C}$; m.f. $\mathrm{C}_{14} \mathrm{H}_{10} \mathrm{~N}_{2} \mathrm{O}_{3} \mathrm{ClF}_{3}$; IR $\left(\mathrm{KBr}, v_{\max }, \mathrm{cm}^{-1}\right)$ : $(\mathrm{C}=\mathrm{O}) 1671$; ${ }^{1} \mathrm{H}$ NMR: $\delta 10.87$ (bs, $1 \mathrm{H}, N H), 9.02-8.96$ (m, 2H, H2', H6'), 8.19 (d, $\left.1 \mathrm{H}, J=9.07 \mathrm{~Hz}, H 5^{\prime}\right), 7.73$ (s, 1H, H6), 6.55 (s, 1H, H3), 3.73 (s, 3H, $\left.-\mathrm{CH}_{3}\right) ;{ }^{13} \mathrm{C}$ NMR: $\delta 166.3,159.7,156.7,150.5,144.9$. 143.1, 130.9, 122.0, 115.7, $114.9(J=263 \mathrm{~Hz}), 113.8,113.5$, 103.1, 55.6.

5-Chloro-2-hydroxy-4-methoxy-N-(2-methylpyridin4-yl)benzamide (4g): m.p. $346-348{ }^{\circ} \mathrm{C}$; m.f. $\mathrm{C}_{14} \mathrm{H}_{13} \mathrm{~N}_{2} \mathrm{O}_{3} \mathrm{Cl}$; IR $\left(\mathrm{KBr}, \mathrm{v}_{\max }, \mathrm{cm}^{-1}\right)$ : $(\mathrm{C}=\mathrm{O}) 1685 ;{ }^{1} \mathrm{H}$ NMR: $\delta 10.73(\mathrm{bs}, 1 \mathrm{H}$, $N H), 8.59$ (d, $\left.1 \mathrm{H}, J=7.63 \mathrm{~Hz}, H 6^{\prime}\right), 7.63-7.52$ (m, 2H, H3', $\left.H 5^{\prime}\right), 7.68$ (s, 1H, H6), 6.47 (s, 1H, H3), 3.76 (s, 3H, $\left.-\mathrm{CH}_{3}\right)$, 2.46 (s, $\left.3 \mathrm{H},-\mathrm{CH}_{3}\right) ;{ }^{13} \mathrm{C} \mathrm{NMR}: \delta 165.8,159.4,157.9,156.3,150.4$, 144.3, 130.8, 114.7, 114.3, 113.9, 112.2, 103.2, 55.3, 24.7.

5-Chloro-N-(2-chloropyridin-4-yl)-2-hydroxy-4methoxybenzamide (5g): m.p. $365-367^{\circ} \mathrm{C}$; m.f. $\mathrm{C}_{13} \mathrm{H}_{10} \mathrm{~N}_{2} \mathrm{O}_{3} \mathrm{Cl}_{2}$; IR $\left(\mathrm{KBr}, \mathrm{v}_{\max }, \mathrm{cm}^{-1}\right)$ : $(\mathrm{C}=\mathrm{O}) 1670 ;{ }^{1} \mathrm{H}$ NMR: $\delta 10.81(\mathrm{bs}, 1 \mathrm{H}$, $N H), 8.72$ (d, 1H, $\left.J=9.38, H 6^{\prime}\right), 8.06-8.03\left(\mathrm{~m}, 2 \mathrm{H}, H 3^{\prime}, H 5^{\prime}\right)$, 7.72 (s, 1H, H6), 6.54 (s, 1H, H3), $3.78\left(\mathrm{~s}, 3 \mathrm{H},-\mathrm{CH}_{3}\right) ;{ }^{13} \mathrm{C}$ NMR: $\delta 165.8,159.1,156.3,156.0,145.3,143.8,131.2,115.0$, 114.3, 113.7, 113.2, 103.0, 55.1.

5-Chloro-N-(3, 5-dichloropyridin-4-yl)-2-hydroxy-4methoxybenzamide (6g): m.p. 386-388 ${ }^{\circ} \mathrm{C}$; m.f. $\mathrm{C}_{13} \mathrm{H}_{9} \mathrm{~N}_{2} \mathrm{O}_{3} \mathrm{Cl}_{3}$; IR $\left(\mathrm{KBr}, v_{\max }, \mathrm{cm}^{-1}\right):(\mathrm{C}=\mathrm{O}) 1654 ;{ }^{1} \mathrm{H}$ NMR: $\delta 10.84$ (bs, $1 \mathrm{H}$, NH), 8.79-8.74 (m, H2',H6'), 7.70 (s, 1H, H6), 6.51 (s, 1H, H3), 3.74 (s, $\left.3 \mathrm{H},-\mathrm{CH}_{3}\right) ;{ }^{13} \mathrm{C}$ NMR: $\delta 166.6,169.4,156.4,149.9$, 142.3, 130.5, 115.5, 114.9, 114.4, 103.6, 55.3. 
2-Hydroxy-4-methoxy-N-(pyridin-4-yl)benzamide (1h): m.p. $279-281{ }^{\circ} \mathrm{C}$; m.f. $\mathrm{C}_{13} \mathrm{H}_{12} \mathrm{~N}_{2} \mathrm{O}_{3}$; IR $\left(\mathrm{KBr}, \mathrm{v}_{\max }, \mathrm{cm}^{-1}\right)$ : $(\mathrm{C}=\mathrm{O}) 1683 ;{ }^{1} \mathrm{H}$ NMR: $\delta 10.89$ (bs, $\left.1 \mathrm{H}, N H\right), 8.55-8.48(\mathrm{~m}$, 2H, H2' $\left.H 6^{\prime}\right), 7.83$ (d, $\left.1 \mathrm{H}, J=8.65 \mathrm{~Hz}, H 6\right), 7.05-6.98(\mathrm{~m}$, 2H, H3', H5'), 6.60-6.57 (m, 2H, H3, H5), 3.80 (s, 3H, $\left.-\mathrm{OCH}_{3}\right)$; ${ }^{13} \mathrm{C}$ NMR: $\delta 167.5,164.1,160.6,150.5,145.5,131.1,116.3$, $114.5,111.9,100.2,55.6$.

N-(3-Chloropyridin-4-yl)-2-hydroxy-4-methoxybenzamide (2h): m.p. 304-306 ${ }^{\circ} \mathrm{C}$; m.f. $\mathrm{C}_{13} \mathrm{H}_{11} \mathrm{~N}_{2} \mathrm{O}_{3} \mathrm{Cl}$; IR $\left(\mathrm{KBr}, \mathrm{v}_{\max }, \mathrm{cm}^{-1}\right)$ : $(\mathrm{C}=\mathrm{O}) 1666 ;{ }^{1} \mathrm{H} \mathrm{NMR}: \delta 10.90$ (bs, $\left.1 \mathrm{H}, \mathrm{NH}\right)$, 8.72 (s, 1H, H2'), 8.61 (d, $\left.1 \mathrm{H}, J=9.60 \mathrm{~Hz}, H 6^{\prime}\right), 7.93(\mathrm{~d}, 1 \mathrm{H}$, $\left.J=9.61 \mathrm{~Hz}, H 5^{\prime}\right), 8.55-8.48\left(\mathrm{~m}, 2 \mathrm{H}, H 2^{\prime}, H 6^{\prime}\right), 7.83$ (d, $1 \mathrm{H}, J$ $=8.65 \mathrm{~Hz}, H 6), 6.59-6.55(\mathrm{~m}, 2 \mathrm{H}, H 3, H 5), 3.82(\mathrm{~s}, 3 \mathrm{H}$, $\left.-\mathrm{OCH}_{3}\right) ;{ }^{13} \mathrm{C}$ NMR: $\delta 167.3,164.0,160.4,150.3,144.4,144.0$, 131.0, 116.4, 123.4, 115.8, 112.1, 100.3, 55.5.

N-(3-(Trifluoromethyl)pyridin-4-yl)-2-hydroxy-4methoxybenzamide (3h): m.p. $290-292{ }^{\circ} \mathrm{C}$; m.f. $\mathrm{C}_{14} \mathrm{H}_{11} \mathrm{~N}_{2} \mathrm{O}_{3} \mathrm{~F}_{3}$; IR $\left(\mathrm{KBr}, \mathrm{v}_{\max }, \mathrm{cm}^{-1}\right)$ : $(\mathrm{C}=\mathrm{O}) 1667 ;{ }^{1} \mathrm{H}$ NMR: $\delta 10.96$ (bs, $1 \mathrm{H}$, $N H)$, 8.97-8.92 (m, 2H, H2', H6'), $8.24(\mathrm{~d}, 1 \mathrm{H}, J=9.65 \mathrm{~Hz}$, $\left.H 5^{\prime}\right), 7.85$ (d, $\left.1 \mathrm{H}, J=9.27 \mathrm{~Hz}, H 6\right), 7.05-6.98$ (m, 2H, H3', $\left.H 5^{\prime}\right), 6.59-6.55$ (m, 2H, H3, H5), $3.79\left(\mathrm{~s}, 3 \mathrm{H},-\mathrm{OCH}_{3}\right) ;{ }^{13} \mathrm{C}$ NMR: $\delta 167.7,164.2,160.7,150.7,146.3,143.5,131.2,124.2$, 121.5 $(J=266 \mathrm{~Hz}), 116.1,114.3,111.8,100.1,55.7$.

2-Hydroxy-4-methoxy-N-(2-methylpyridin-4-yl)benzamide (4h): m.p. $285-287^{\circ} \mathrm{C}$; m.f. $\mathrm{C}_{14} \mathrm{H}_{14} \mathrm{~N}_{2} \mathrm{O}_{3}$; IR (KBr, $v_{\max }$, $\left.\mathrm{cm}^{-1}\right)$ : $(\mathrm{C}=\mathrm{O}) 1691 ;{ }^{1} \mathrm{H}$ NMR: $\delta 10.82(\mathrm{bs}, 1 \mathrm{H}, N H), 8.59(\mathrm{~d}, 1 \mathrm{H}$, $\left.J=8.23 \mathrm{~Hz}, H 6^{\prime}\right), 7.05-6.97$ (m, 2H, H3', H5'), 7.80 (d, 1H, $J=$ $8.68 \mathrm{~Hz}, J=1.77 \mathrm{~Hz}, H 6), 6.65-6.52$ (m, $1 \mathrm{H}, H 4), 3.80$ (s, 3H, $\left.-\mathrm{OCH}_{3}\right) ;{ }^{13} \mathrm{C}$ NMR: $\delta 167.6,164.2,160.6,154.2,150.6,144.8$, $131.1,116.1,114.9,113.0,111.8,100.2,55.8,24.2$.

N-(2-Chloropyridin-4-yl)-2-hydroxy-4-methoxybenzamide (5h): m.p. 181-183 ${ }^{\circ} \mathrm{C}$; m.f. $\mathrm{C}_{13} \mathrm{H}_{11} \mathrm{~N}_{2} \mathrm{O}_{3} \mathrm{Cl}$; IR $\left(\mathrm{KBr}, v_{\max }, \mathrm{cm}^{-1}\right):(\mathrm{C}=\mathrm{O}) 1674 ;{ }^{1} \mathrm{H}$ NMR: $\delta 10.90(\mathrm{bs}, 1 \mathrm{H}, \mathrm{NH})$, $8.74\left(\mathrm{~d}, 1 \mathrm{H}, J=9.45, H 6^{\prime}\right), 8.08-8.06$ ( $\left.\mathrm{m}, 2 \mathrm{H}, H 3^{\prime}, H 5^{\prime}\right), 7.84$ $(\mathrm{d}, 1 \mathrm{H}, J=8.57 \mathrm{~Hz}, H 6), 6.62-6.58$ (m, 2H, H3, H5), 3.82 (s, $\left.3 \mathrm{H},-\mathrm{OCH}_{3}\right) ;{ }^{13} \mathrm{C}$ NMR: $\delta 167.9,164.3,160.7,153.6,145.8$, 144.3, 131.2, 116.2, 115.9, 114.8, 111.7, 100.0, 55.8.

N-(3, 5-Dichloropyridin-4-yl)-2-hydroxy-4-methoxybenzamide (6h): m.p. 224-226 ${ }^{\circ} \mathrm{C}$; m.f. $\mathrm{C}_{13} \mathrm{H}_{10} \mathrm{~N}_{2} \mathrm{O}_{3} \mathrm{Cl}_{2}$; IR $\left(\mathrm{KBr}, \mathrm{v}_{\max }, \mathrm{cm}^{-1}\right)$ : $(\mathrm{C}=\mathrm{O}) 1659 ;{ }^{1} \mathrm{H} \mathrm{NMR}: \delta 10.93$ (bs, $\left.1 \mathrm{H}, \mathrm{NH}\right)$, 8.81-8.77 (m, 2H, H2', H6'), 7.82 (d, 1H, $J=8.58 \mathrm{~Hz}, H 6$ ), $6.59-6.55$ (m, 2H, H3, H5), 3.77 (s, 3H, $\left.-\mathrm{OCH}_{3}\right) ;{ }^{13} \mathrm{C} \mathrm{NMR}$ : \$167.1, 163.8, 159.7, 149.6, 142.7, 129.7, 124.3, 116.6, 112.1, $100.5,55.8$

Biological Activity: The following strains, obtained from Pulmonary Hospital of Lanzhou, were used for the evaluation of in vitro antimycobacterial activity: Mycobacterium tuberculosis PHL 474/528 and Mycobacterium avium PHL 599/ 778. Antimycobacterial activity of the compounds against these strains was determined in the Sauton semiliquid medium (with $5 \%$ bovine serum). The Sauton semiliquid medium was routinely used in Pulmonary Hospital of Lanzhou. Each strain was simultaneously inoculated into a Petri dish containing the improved Löwenstein-Jensen medium for the control of the sterility of the inoculums and its growth ${ }^{15,16}$. The compounds were added to the medium in sodium phosphate buffer solutions with 7.2 of $\mathrm{pH}$ value. The final concentrations were 1200 , $600,300,150,75,37.5,18.8,9.4,4.7,2.3$ and $1.2 \mu \mathrm{mol} \mathrm{L}^{-1}$. MICs, at which inhibition of the growth of the mycobacteria occurred, were determined after incubation at $37{ }^{\circ} \mathrm{C}$ for 4 weeks (Table-1).

\section{RESULTS AND DISCUSSION}

The salicylanilides could inhibit the TCS nonspecifically by a mechanism involving in uncoupling oxidative phosphorylation ${ }^{6}$. With respect to the activities against $M$. tuberculosis (TB), there are two additional major aspects considered: innate resistance, the unique structure and composition of the highly hydrophobic cell wall that must be traversed. It is well known that increasing the lipophilicity improve cell wall permeability ${ }^{12-14}$, which implied that the differences in antimicrobacterial activities of N-pyridin-4-yl salicylamide analogues arises from the differences in lipophilicity. Furthermore, we studied the influence of the substituent effect on the heterocycle and salicylic moieties. The minimum inhibitory concentrations (MICs) against $M$. avium (A) of the eight substrates (2c, $\mathbf{4 c}$, 5c, 4e, 5e, 2d, 4f) are equal to $75 \mu \mathrm{mol} \mathrm{L}{ }^{-1}$, which are remarkably lower than $300 \mu \mathrm{mol} \mathrm{L}{ }^{-1}$ of the MIC against A of isoniazide. Among them, compounds $\mathbf{4 c}, \mathbf{5 c}, \mathbf{4 e}$ and $\mathbf{5 e}$ exhibit good antiTB activity compared to isoniazide (Table-1). These results suggest that such compounds might serve as prospective widespectrum antimycobacterial substances.

Table- 1 showed that when position 2 of the heterocycle, or position 3 or 5 of the salicylic moiety was substituted by chlorine, methyl or bromine, the antimycobacterial activity will be increased. This antimycobacterial value is particularly high against the typical mycobacterial strains A, For example, the compounds $\mathbf{4 c}, \mathbf{5 c}, \mathbf{4 e}$ and $\mathbf{5 e}$ in anti-TB activity are almost the same as INH. However, they exhibit higher anti-A activity than INH. Therefore, it is likely that N-(pyridin-4-yl) salicylamides may constitute a new group of promising compounds bearing potential antimycobacterial activity.

When higher hydrophobicity compounds substituted on position 2 of the heterocyclic moiety were used, the antimycobacterial activity decreased dramatically (e.g. 4c, 5c, 4e and 5e). While introduction of methoxyl group on position 4 of the salicylic moiety, with lower hydrophobicity, led to decreased activity. In general, introduction of higher hydrophobic substituents leads to higher activity towards both anti-TB and anti-A. However, based on our research, the activities were affected not only by hydrophobicity but also structure and electronic requirements. For example, 5c and 5e with $\lg P$ more than 2.5 resulted in high potency, but $\mathbf{6 b}$ and $\mathbf{6 e}$ with $\lg \mathrm{P}$ more than 3 gave decreased potency.

The compounds substituted on position 3 and 5 of the heterocyclic moiety and on position 3 of the salicylic moiety exhibited decreased antimycobacterial activity in spite of higher or lower hydrophobicity. These results might be so due to the steric reasons for the substituents can prevent the donor or acceptor of amide and hydroxyl groups from interacting with the corresponding biomacromolecule by hydrogen bond.

\section{Conclusion}

Present studies of structure-activity relationship suggests that the introduction of substitutes with some balance between hydrophobicity and electron effects in position 2 of the heterocyclic, or in position 3 or 5 of the salicylic moiety may greatly affect the antimycobacterial activity, which contribute to the 


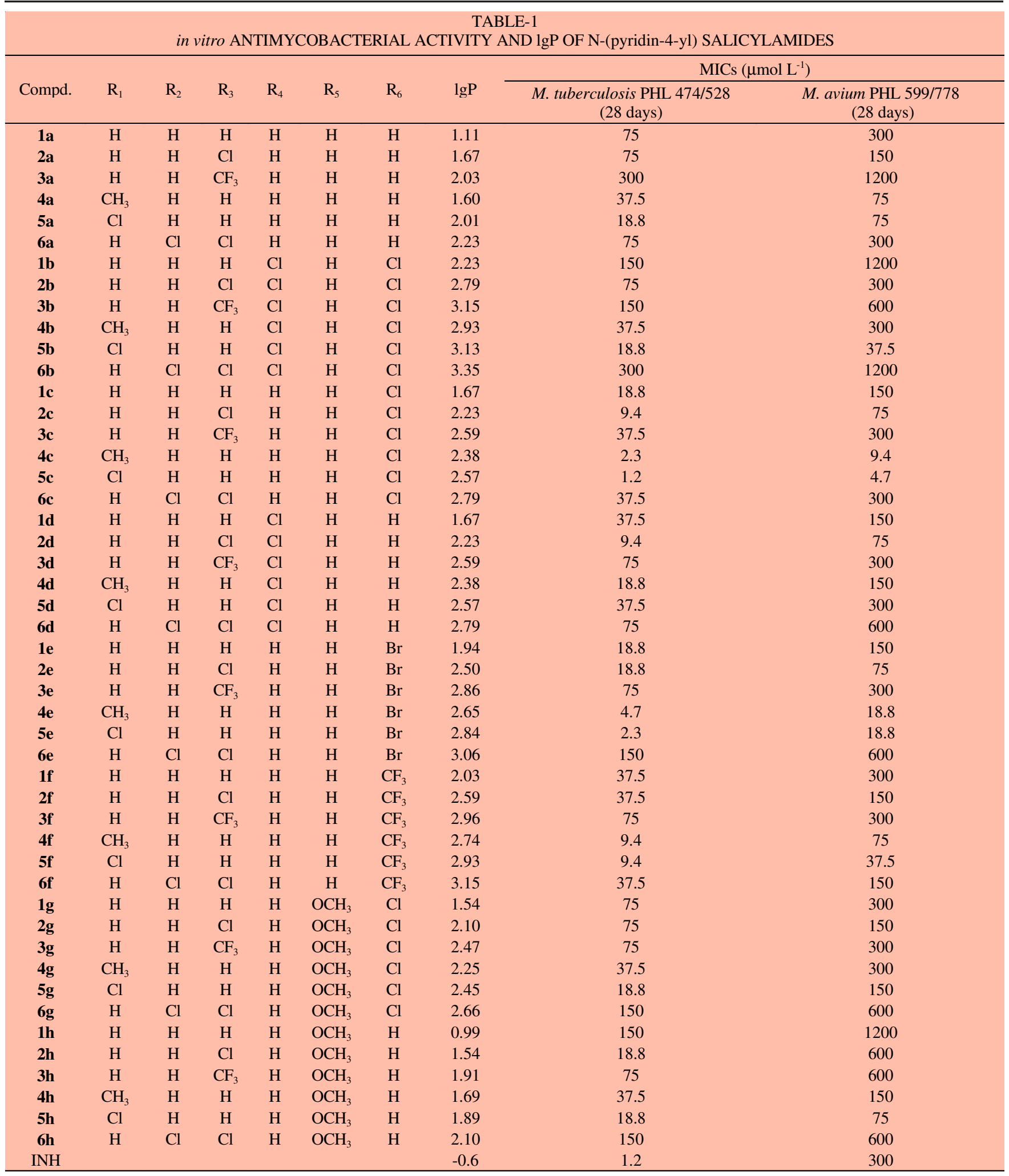

discovery of a series of prospective wide-spectrum antimycobacterial substances. The applications of these results and further investigations based on such methodology are underway in our laboratory.

\section{ACKNOWLEDGEMENTS}

The authors are grateful to Pulmonary Hospital of Lanzhou for the financial support of this research.

\section{REFERENCES}

1. K. Waisser, J. Hladuvkova, J. Kunes, L. Kubicova, V. Klimesova, P. Karajannis and J. Kaustova, Chem. Pap., 55, 121 (2001).

2. K. Waisser, O. Bures, P. Holý, J. Kunes, R. Oswald, L. Jirásková, M. Pour, V. Klimesová, L. Kubicová and J. Kaustová, Arch. Pharm., 336, 53 (2003). 3. D.J. Hlasta, J.P. Demers, B.D. Foleno, S.A. Fraga-Spano, J. Guan, J.J. Hilliard, M.J. Macielag, K.A. Ohemeng, C.M. Sheppard, Z. Sui, G.C. Webb, M.A. Weidner-Wells, H. Werblood and J.F. Barrett, Bioorg. Med. Chem. Lett., 8, 1923 (1998). 
4. J.B. Stock, A.J. Ninfa and A.M. Stock, Microbiol. Rev., 53, 450 (1989).

5. J.A. Hoch and T.J. Silhavy, Two-Component Signal Transduction, ASM Press: Washington, DC, pp 305-317 (1995).

6. R.A. Goldschmidt, M.J. Macielag and D.J. Hlasta, Curr. Pharm. Des., 3, 125 (1997).

7. M.J. Macielag, J.P. Demers, S.A. Fraga-Spano, D.J. Hlasta, S.G. Johnson, R.M. Kanojia, R.K. Russell, Z. Sui, M.A. Weidner-Wells, H. Werblood, B.D. Foleno, R.M. Goldschmidt, M.J. Loeloff, G.C. Webb and J.F. Barrett, J. Med. Chem., 41, 2939 (1998).

8. I.M. Ota and A. Varshavsky, Science, 262, 566 (1993).

9. K. Waisser, M. Pesina, P. Holý, M. Pour, O. Bures, J. Kunes, V. Klimesová, V. Buchta, P. Kubanová and J. Kaustová, Arch. Pharm., 336, 322 (2003).

10. K. Waisser, M. Perina, V. Klimešová and J. Kaustová, Coll. Czech. Chem. Commun., 68, 1275 (2003).
11. K. Waisser, K. Drazková, J. Kunes, V. Klimesová and J. Kaustová, IL Farmaco, 59, 615 (2004).

12. F. Doucet-Populaire, J.O. Capobianco, D. Zakula, V. Jarlier and R.C. Goldman, J. Antimicrob. Chemother., 41, 179 (1998).

13. R.C. Goldman and F. Scaglione, Curr. Drug Targets Infect. Disord., 4, 241 (2004).

14. Z.J. Zhu, O. Krasnykh, D. Pan, V. Petukhova, G. Yu, Y. Liu, H. Liu, S. Hong, Y. Wang, B. Wan, W. Liang and S.G. Franzblau, Tuberculosis, 88(Suppl. 1), 549 (2008).

15. M. Botta, M. Artico, S. Massa, A. Gambacorta, M.E. Marongiu, A. Pani and P. Lacolla, Eur. J. Med. Chem., 27, 251 (1992).

16. Chinese Anti-tuberculosis Association, J. Chinese Anti-tuberc. Assoc., 18, 28 (1996) 